\title{
UTILISATION DU LOGICIEL CALYPSO LORS DE LA RECONSTRUCTION DU POSTE 9 AU PORT AUTONOME INTERNATIONAL DE DJIBOUTI
}

Roland Boutin

Chef de la division études au service technique des travaux immobiliers et maritimes, 15 rue de Laborde, 00309 ARMEES

Luc Barbot

Responsable du département Génie Civil, société EEG SIMECSOL, agence de Nantes, Le Ponant2 - Zone Atlantis, 44812 Saint Herblain Cedex

André Emaille

Directeur de l'export, société ETPO, 3 place du Sanitat, 44100 Nantes Cedex

\section{Résumé}

Le Projet National TUBA lancé par l'LREX' permet de disposer aujourd'hui d'une procédure complète de prévision et de contrôle du battage des pieux et profilés métalliques intégrée au sein du logiciel CALYPSO. Celle-ci a été mise en œuvre lors du battage des 44 pieux métalliques nécessaires à la reconstruction du poste 9 au port autonome international de Djibouti.

Cette procédure a permis de valider la faisabilité du chantier de battage. Elle a confirmé l'adéquation des moyens prévus avant leur envoi sur site. Grâce à l'instrumentation en cours de battage réalisée sur deux pieux en début de chantier, il a été possible de réévaluer les paramètres de sol et ainsi de préciser la fiche minimale à atteindre et les critères d'arrêt pour chaque file de pieux.

\section{Abstract}

TUBA French project, launched by IREX Institute, allows to have a complete procedure for planning and checking of driving of piles and steel-profiles. The procedure is integrated within the CALYPSO software. This procedure was set up during the driving of 44 steel piles for the building of the new berth 9 in Djibouti international harbour.

This procedure allows us to confirm the feasibility of the pile driving. The method consists in predicting the use of the means needed for pile driving, before they are used on site. The prediction is confirmed by instrumenting two piles at the beginning of works on site, therefore allowing the real measurement of ground data, and therefore giving a more accurate minimum target penetration value, and a more accurate value for the acceptability of each line of piles.

\footnotetext{
${ }^{1}$ Institut pour la Recherche appliquée et l'EXpérimentation en génie civil
} 


\section{Introduction}

La recherche française dans le domaine du génie civil trouve au travers de l'IREX une structure fédératrice permettant à des acteurs tant privés que publics de joindre leurs efforts pour faire avancer les techniques. Le projet national TUBA en est une illustration dans le domaine des travaux portuaires. Avec le soutien de la DRAST ${ }^{2}$, une procédure complète de prévision et de contrôle du battage des pieux et profilés métalliques a en effet été développée. Nous allons illustrer ciaprès ce qu'apporte cette démarche au travers du cas concret de la rénovation du poste 9 au port autonome international de Djibouti.

\section{Présentation générale du chantier et du site}

La reconstruction du poste 9 au port international de Djibouti consistait à réaliser un appontement de $70 \mathrm{~m}$ de long pour $20 \mathrm{~m}$ de large après démolition des ouvrages existants. En partie arrière, un terre-plein de stockage conteneurs d'environ $4000 \mathrm{~m}^{2}$ a été réalisé (figure 1). Le nouveau quai offre un tirant d'eau de $9 \mathrm{~m}$. Le coût des travaux s'élève à environ $27 \mathrm{MF}$. L'installation a été inaugurée en décembre 1998.

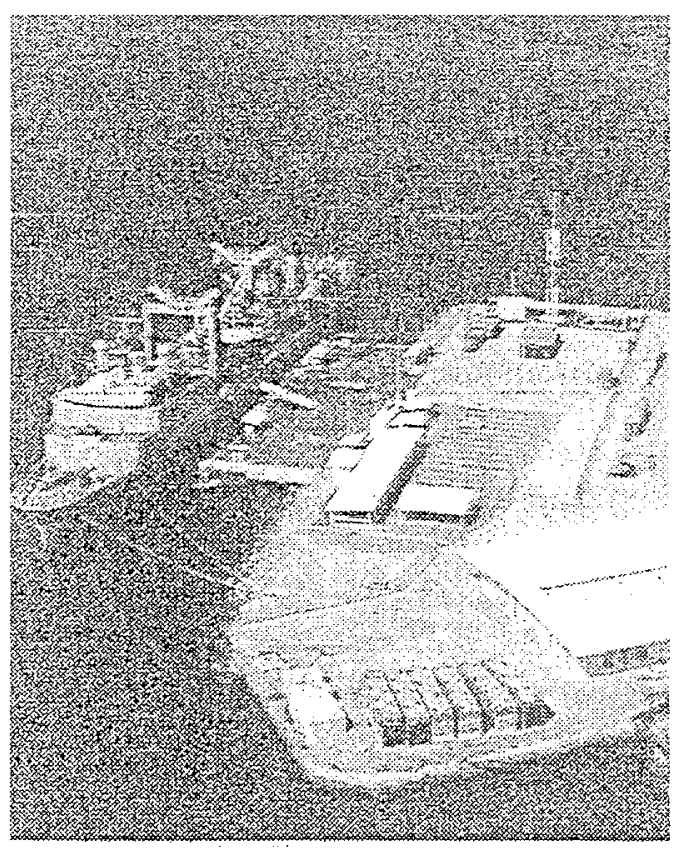

Figure 1 : Fue générale / general view

Afin de tenir compte du risque sismique, l'ouvrage a été dimensionné pour des conditions sismiques de zone III au sens du zonage afférent au territoire français. Cette contrainte s'est avérée très dimenșionnante tant pour les taux de ferraillage des éléments en béton armé que pour les efforts sur les pieux. Comme le montre la coupe type de. l'ouvrage (figure 2), il a également fallu tenir compte de la présence de l'épave du Fontainebleau dans le terre-plein.

\footnotetext{
${ }^{2}$ Direction de la Recherche et des Affaires Scientifiques et Techniques du ministère de l'Equipement:
} 


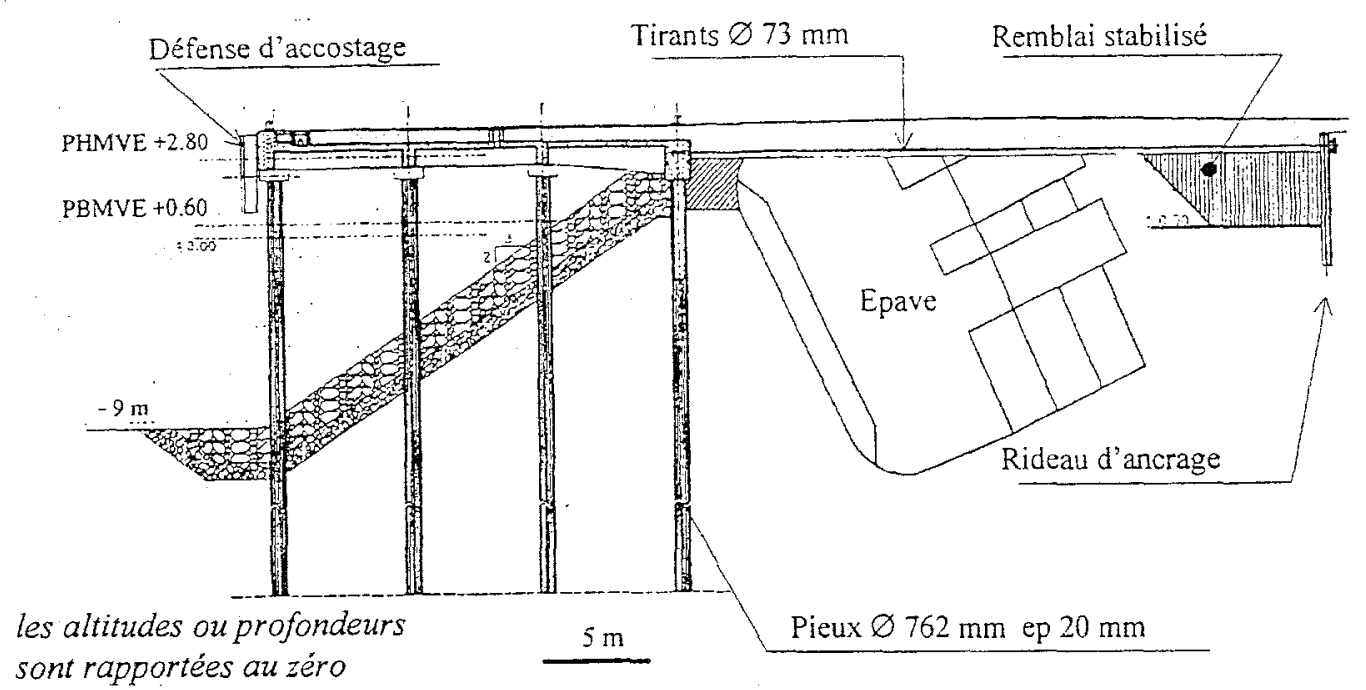

portées au zéro hydrographique (zéro hydro)

Figure 2 : Coupe type/sectional view

Différentes campagnes géotechniques réalisées sur le site permettent d'avoir une bonne idée des conditions de sol. Un profil géotechnique simplifié est donné sur la figure 3 . L'appontement est constitué de 44 pieux tubes métaliliques de $762 \mathrm{~mm}$ de diamètre et de $20 \mathrm{~mm}$ d'épaisseur ayant une limite élastique de $420 \mathrm{MPa}$. Ils sont mis en œuvre ouverts à leur base. Après mise en place par vifrofonçage, ils ont été battus de plusieurs mètres dans la couche d'argile compacte et madrépores qui présente des caractéristiques mécaniques élevées. Le battage a été réalisé à l'aide d'un marteau diesel de type DELMAG D25/32 muni d'un martyr en bois AZOBE de $170 \mathrm{~mm}$ d'épaisseur. Les études de sols laissaient apparaître que le toit de cet horizon pouvait comporter une frange légèrement altérée sur des épaisseurs de 6 à 8 mètres.

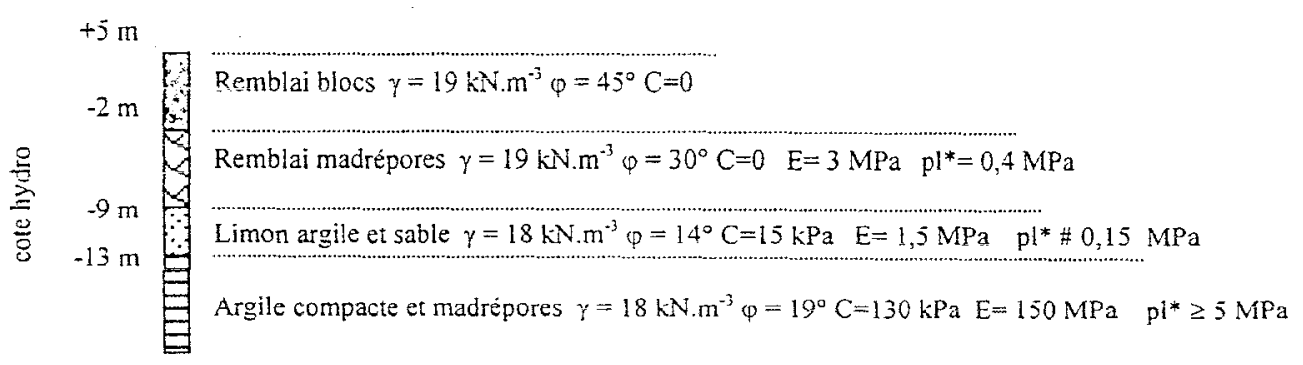

Figure 3 : Profil géotechnique simplifié / simplified soil profile

La mise en œuvre des pieux a été suivie à l'aide de la procédure disponible dans le logiciel CALYPSO 3 développée dans le cadre du projet national TUBA.

${ }^{3}$ Calcul informatique intégré des pieux de structures océaniques 


\section{La procédure Calypso, présentation}

La méthodologie développée dans CALYPSO est une adaptation des pratiques offshore aux travaux portuaires. L'architecture du système est schématisée sur la figure 4.

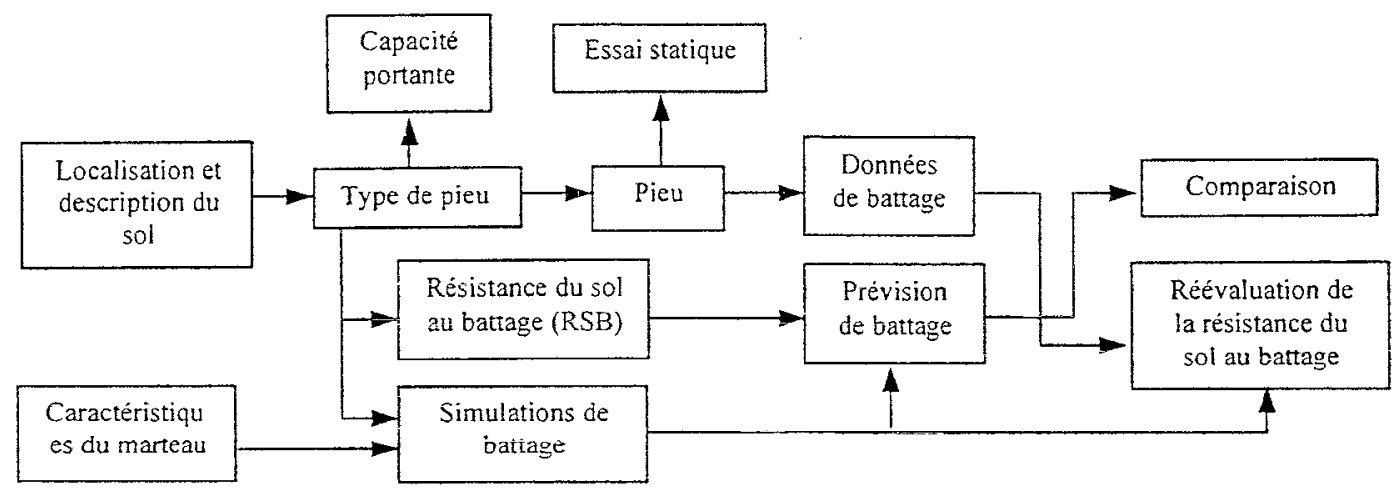

Figure 4: Architecture de CALYPSO / CALYPSO architeciure

Les différents modules de calculs permettent de suivre un projet tout au long de son évolution. Au stade de la conception, le module capacité portante est utilisé. Différentes méthodes sont disponibles (fascicule 62 titre $5, \mathrm{API}^{4}, \ldots$ ). Le module prévision de battage permet d'établir les courbes donnant le nombre de coups par $25 \mathrm{~cm}$ d'enfoncement en fonction de la profondeur de pénétration. Pour cela les modules résistance du sol au battage $\left(\mathrm{RSB}^{5}\right)$ et simulation de battage sont utilisés. Cette prévision permettra d'une part de valider la faisabilité du projet et d'autre part de définir les procédures et matériels à utiliser sur le chantier. Une fois sur le chantier, une instrumentation des pieux permet de mesurer les accélérations et les contraintes induites dans la tête du pieu lors du battage. L'analyse des données obtenues permet de valider les méthodes prévisionnelles de battage ou les paramètres utilisés et à défaut de les modifier afin d'obtenir une modélisation plus représentative des données mesurées. Cette démarche est particulièrement utile pour la simulation de la RSB pour laquelle le module réévaluation de la $\mathrm{RSB}$ au battage est utilisé. Une fois cette démarche terminée, il est possible de conclure sur les critères d'arrêt de battage à suivre pour le reste du chantier.

Pour obtenir davantage de détails théoriques et pratiques, le lecteur pourra se reporter à l'article de Vergobbi et Poulet (1992) et aux rapports de Borel et al (1994) et de Dendani et al (1994) établis pour le projet national Tuba.

\footnotetext{
${ }^{4}$ American Petroleum Institute

5ou SRD de l'anglais "soil resistance to driving"
} 


\section{La procédure Calvpso, cas de la reconstruction du poste 9 à Djibouti}

\subsection{La prévision de battage}

A partir des données de sol et des caractéristiques des pieux à mettre en œuvre, les faisceaux prévisionnels de RSB en fonction de la pénétration ont été estimés en appliquant les recommandations de Stevens et al (1982) et de Semple et al (1981). Des simulations de battage ont alors été effectuées pour différentes profondeurs et pour des rendements de marteau de 20,30 et 40\%. Les résultats s'expriment en terme de RSB en fonction du nombre de coups par enfoncement de $25 \mathrm{~cm}$. Ces simulations permettent d'estimer la contrainte dynamique induite dans le tube par le battage. La valeur maximale obtenue était de $147 \mathrm{MPa}$ pour un rendement de $40 \%$ ce qui est très inférieur au $2 / 3$ de la limite élastique de l'acier des tubes.

La comparaison des courbes de simulation et des courbes de RSB permet enfin de déduire les courbes de nombre de coups par unité d'enfoncement en fonction de la pénétration du pieu. C'est la prévision de battage. La simulation obtenue pour un rendement compris entre 30 et $40 \%$ est donnée figure 10 .

Cette prévision de battage aura permis d'une part de s'assurer que l'intégrité des pieux pouvait être respectée au cours du battage et d'autre part de valider l'adéquation des moyens de battage prévus. Ce dernier point est particulièrement sensible pour un chantier à l'export.

\subsection{L'instrumentation}

Les deux premiers pieux du chantier ont été instrumentés en cours de battage (figure 5). L'équipement installé en tête de pieu est composé de deux capteurs combinés diamétralement opposés. Ces capteurs sont constitués chacun d'un accéléromètre piézo-résistif et d'une jauge de déformation (mesure de la contrainte). Un boitier de jonction permet la connexion entre les capteurs et le câble de liaison avec la centrale dacquisition (figures 6 et 7 ). Les capteurs sont disposés à environ $1.4 \mathrm{~m}$ sous la tête du pieu de façon à se placer dans une zone où la propagation des ondes puisse être considérée comme unidimensionnelle. La mise en place des capteurs et de la boîte de jonction du câble de transmission est effectuée sur le pieu déjà en place et après vibrofonçage.

L'analyse des mesures sur site permet de traiter l'ensemble des coups de marteau et de visualiser, en temps réel sur l'écran, les signaux de contrainte et de vitesse (figure 8).

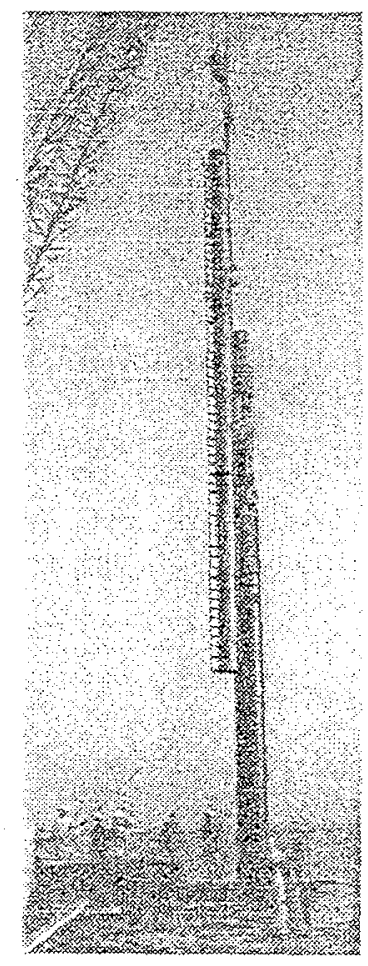

Figure 5: Battage du pieu D1 / DI pile driving 


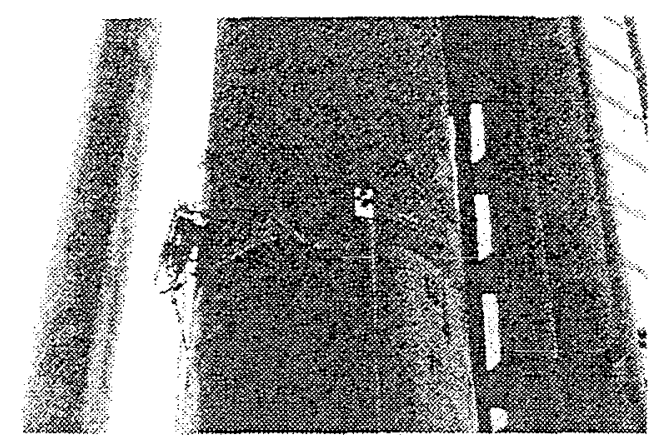

Figure 6 : Pieu instrumenté $I$ instrumented pile

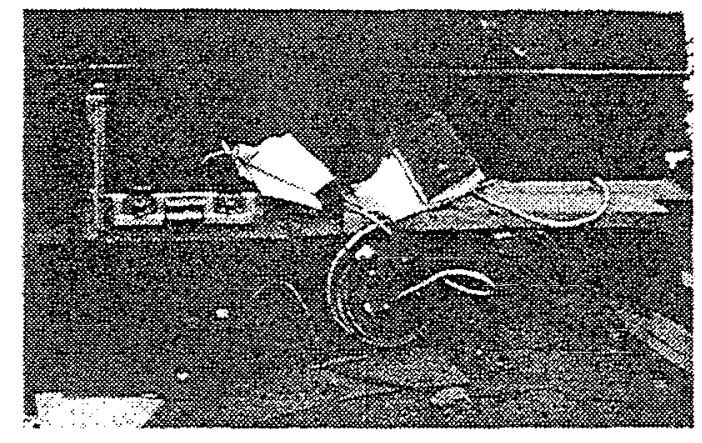

Figure 7 : Centrale d'acquisition/ data recording computer

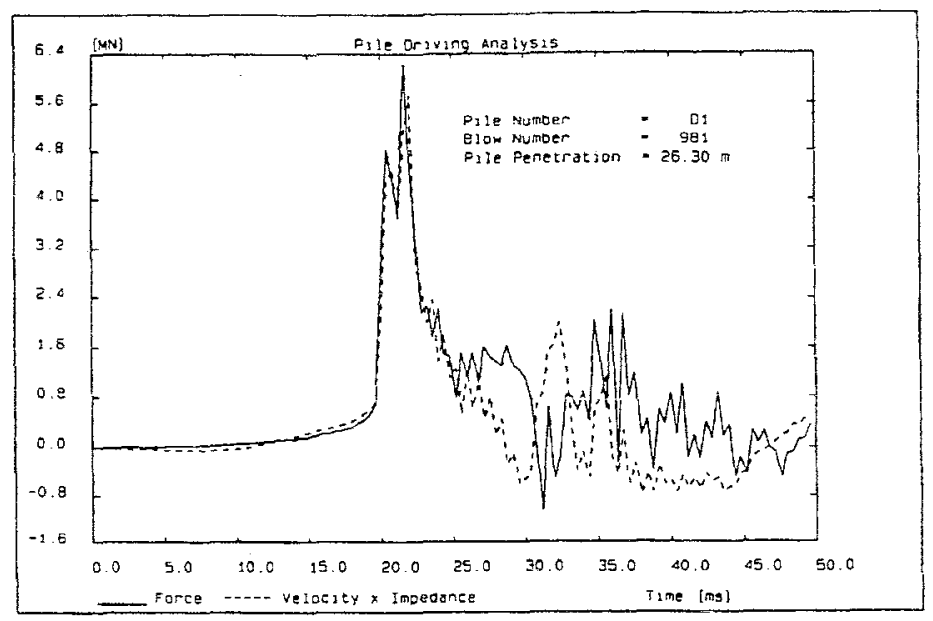

Figure 8 : Exemple de mesures / recorded measurements

De nombreux paramètres sont consultés et visualisés à l'écran en temps réel pendant le battage :

- la contrainte due à l'impact en tête de pieu,

- l'énergie nette délivrée au pieu,

- l'énergie globale du système de battage (rendement), exprimée par le rapport de l'énergie nette délivrée au pieu (mesurée) à l'énergie nominale délivrée par le marteau (fournie par le constructeur),

- le taux de battage exprimé en nombre de coups pour $25 \mathrm{~cm}$ d'enfoncement,

- l'excentricité du choc évaluée par le rapport des pics de contrainte mesurés par les deux jauges de déformation diamétralement opposées.

Ces informations permettent de :

- vérifier les performances réelles du marteau et de les comparer aux performances prises en compte dans les analyses prévisionnelles de battage ;

- contrôler en permanence le niveau de contrainte dans le pieu et réduire ainsi les risques d'endommagement de sa pointe sur un horizon dur.

La contrainte de compression dynamique est directement mesurée par les capteurs de jauges mis en place en tête de pieu. Les contraintes maximales mesurées en 
tête de pieu ont varié au cours du battage entre 100 et $160 \mathrm{MPa}$, valeurs proches de celles estimées lors de l'étude prévisionnelle.

Le rendement du marteau, mesuré in situ, a été de l'ordre de $38 \%$ en fin de battage. Cette bonne valeur pour un marteau diesel s'explique certainement du fait que le battage est vertical et que les pieux sont de forte section ce qui favorise un bon transfert d'énergie.

L'analyse immédiate des mesures sur chantier a donc confirmé que le battage ne détériorait pas les pieux et a fourni une estimation précise du rendement du marteau. Cette instrumentation aurait également permis de détecter tout comportement "anormal" du sol, aidant ainsi à l'analyse technique d'éventuelle difficulté.

\subsection{Analyse des enregistrements, réévaluation des paramètres de sol}

La courbe de battage (figure 9) donne le taux de battage (nombre de coups nécessaires pour faire pénétrer le pieu de $25 \mathrm{~cm}$ ) en fonction de la profondeur de pénétration. Cette courbe dépend de l'énergie transmise au pieu par le marteau, de la RSB et des paramètres dynamiques du sol en frottement et en pointe. Si ces derniers sont fixés, la connaissance de l'énergie réellement transmise au pieu permet d'estimer la RSB réelle dite encore RSB "mesurée". Pour les lois de comportement retenues dans CALYPSO (Dendani et al (1994)), la réponse dynamique du sol est caractérisée par les facteurs d'amortissement dynamique (j

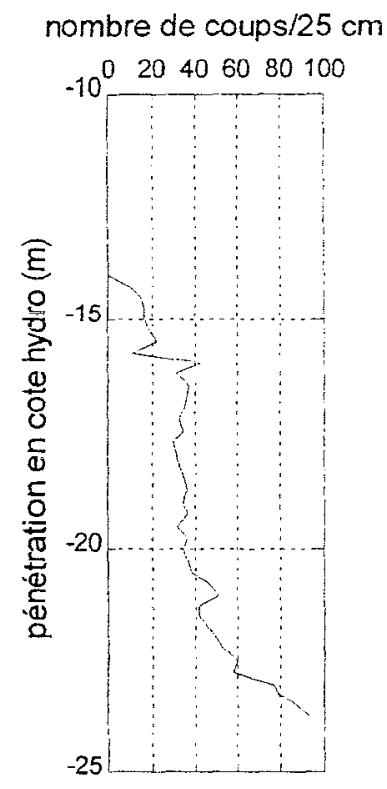

Figure 9 : Courbe de battage / driving curve

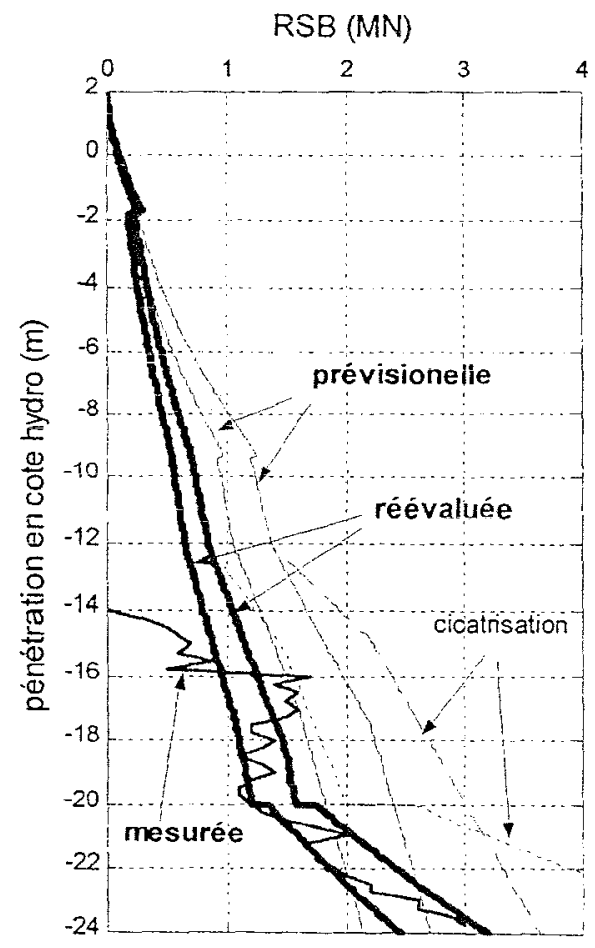

Figure 10 : Courbes de RSB SRD curves 
et $\left.\mathrm{j}_{\mathrm{p}}\right)^{6}$ et les termes de rebond élastique ou "Quake" $\left(\mathrm{Q}_{1} \text { et } \mathrm{Q}_{\mathrm{p}}\right)^{6}$. Les valeurs retenues pour nos calculs (y compris ceux de la simulation de battage initiale) sont $\mathrm{j}_{l}=0.2 \mathrm{~s} / \mathrm{m} ; \mathrm{j}_{\mathrm{p}}=0.5 \mathrm{~s} / \mathrm{m}$ et $\mathrm{Q}_{l}=\mathrm{Q}_{\mathrm{p}}=2.54 \mathrm{~mm}$ conformément aux recommandations de Roussel (1979).

L'instrumentation du pieu D1 permet d'accéder à l'énergie réellement transmise au pieu par le marteau. Il est donc possible de tracer la courbe de RSB "mesurée" en fonction de la profondeur de pénétration (figure 10).

La comparaison entre le faisceau de RSB prévisionnelle établie lors de l'étude prévisionnelle de battage à partir des caractéristiques géomécaniques issues des campagnes géotechniques avec la RSB "mesurée" fait apparaître une tendance générale à la surévaluation de la RSB prévisionnelle jusqu'à environ $-20 \mathrm{~m}$ Hydro. Au-delà, la RSB prévisionnelle a tendance à être sous-évaluée. Cette analyse a conduit au réexamen des études de sols et à la réévaluation des paramètres initialement retenus. La couche d'argile compacte et madrépores a notamment été mieux représentée en distinguant un horizon entre -13 et $-20 \mathrm{~m}$ ayant une cohésion de $90 \mathrm{kPa}$ et un horizon entre -20 et $-26 \mathrm{~m}$ ayant une cohésion croissante de 250 à $350 \mathrm{kPa}$. Ces valeurs qui n'ont pas été initialement retenues pour le profil géotechnique simplifié (figure 3 ) sont plus conformes aux mesures réalisées lors des essais de sols.

Comme le montre la figure 10 , cette phase de réévaluation conduit à un faisceau de RSB simulée réévaluée beaucoup plus conforme à la RSB "mesurée".

\subsection{Justification de la capacité portante des pieux}

Les calculs menés par la société ETPO conduisaient à une capacité portante ultime à reprendre de l'ordre de $3 \mathrm{MN}$ pour la file de pieux la plus chargée.

Pour cette charge, la méthode de dimensionnement pressiométrique du fascicule 62 titre $\mathrm{V}$ préconisait un ancrage de 1 à 2 mètres dans les argiles compactes (cote - 14 à $-15 \mathrm{~m}$ hydro). Or la RSB "mesurée" à - $16 \mathrm{~m}$ hydro est au plus de $1.7 \mathrm{MN}$. Si l'on peut espérer une amélioration de la capacité portante à long terme par cicatrisation, il n'était pas possible de s'arrêter au dimensionnement pressiométrique compte tenu du niveau des efforts à reprendre.

Cette analyse a été renforcée par le fait que la cicatrisation a été très longue à se mobiliser. Sur les différents pieux sur-battus au cours du chantier, l'amélioration de la cicatrisation dans les 15 premiers jours suivant le battage a été très variable. Il est en fait apparu que pour les sols en présence, la cicatrisation, exprimée comme le rapport du nombre de coups pour $25 \mathrm{~cm}$ d'enfoncement lors du surbattage à la valeur obtenue pour le battage des 25 derniers $\mathrm{cm}$ battus initialement, pouvait atteindre $200 \%$ au-delà de 45 jours de repos.

Les études de sol avaient de surcroît mis en évidence un risque d'altération du toit de la couche d'argile compacte et madrépores sur 6 à $8 \mathrm{~m}$ d'épaisseur.

${ }_{6}^{6}$ l'indice l caractérise le terme de frottement latéral et l'indice p celui de l'effort de pointe 
Pour tenir compte de ces différentes observations, la capacité portante statique des pieux a été justifiée en considérant les paramètres géotechniques réévalués issus de l'analyse des données des battages instrumentés. Différentes approches basées sur les méthodes API86 et API93 ont alors été menées. Elles ont conduit à des fiches minimales à respecter de $-22,5 \mathrm{~m}$ hydro pour les files de pieux avant et arrière et de $-24 \mathrm{~m}$ hydro pour les files intermédiaires.

\subsection{Définition des critères d'arrêt '}

En fonction des fiches minimales calculées précédemment, et des résultats du suivi de battage, les critères d'arrêt de battage pour la suite du chantier ont été arrêtés.

Pour les files de pieux avant et arrière, il a été retenu que les pieux devaient être battus au moins jusqu'à la fiche $-22,5 \mathrm{~m}$ hydro et qu'ils pouvaient être acceptés à cette cote uniquement si la cadence de battage mesurée en condition de battage continu et pour un marteau fonctionnant à son énergie maximale, était supérieure ou égale à 45 coups $/ 25 \mathrm{~cm}$ sur une volée de $25 \mathrm{~cm}$ d'enfoncement.

Pour les files intermédiaires, une fois la fiche de - $24 \mathrm{~m}$ hydro atteinte, l'acceptation du pieu était subordonnée à une cadence supérieure ou égale à 75 coups $/ 25 \mathrm{~cm}$ mesuré comme précédemment.

L'application d'une formule de battage traditionnelle comme celle de Kümmel (1985) aurait conduit à un critère d'arrêt de l'ordre du millimètre par coup. Ce résultat confirme que cette approche n'était pas applicable pour notre chantier. Comme le précise Davidian (1969), les formules de battage ne sont en fait pas applicables en terrain argileux qui présente un refus faible (cas des argiles compactes). Cette remarque confirme l'intérêt de la méthode développée dans le cadre du projet TUBA.

\section{Conclusion}

L'utilisation du logiciel CALYPSO couplée à une instrumentation en cours de battage a permis de bien appréhender les caractéristiques géotechniques et de préciser des fiches minimales à prévoir pour chaque file de pieux.

Les moyens à mettre en cuvre sur le chantier ont pu être validés avant l'envoi du matériel sur place. Ce point a été particulièrement appréciable pour un chantier à l'export.

La méthodologie développée dans CALYPSO a permis de définir des critères d'arrêt de battage réalistes. Comme nous l'avons indiqué, les formules de battage classiques auraient été mises en défaut pour notre projet qui concerne des pieux frottants, battus dans des horizons d'argiles compactes.

Enfin, cette procédure permet une approche technique rationnelle du chantier de battage. Elle facilite ainsi grandement les échanges entre la maitrise d'œuvre et l'entreprise chargée de réaliser les travaux. 


\section{Références}

Borel D., Vergobbi P., Puech A. (1994). IREX - Club travaux portuaires - Projet national TUBA - Etudes bibliographiques. GEODIA, rapport 94.16.01, 59 p.

Davidian Z (1969). Pieux et fondations sur pieux. Eyrolles: Paris. $192 \mathrm{p}$.

Dendani H., Vergobbi P., Puech A. (1994). IREX - Club travaux portuaires Projet national TUBA - Etude théorique. GEODIA, rapport 94.16.01, 33 p.

Kümmel F (1985). The Kümmel method for calculation of impact forces in Piles. $2^{\text {nd }}$ International Conference on the Application of Stress-Wave Theory on Piles, Stockholm.

Roussel H.J. (1979). Pile driving analysis of large diameter high capacity offshore pipe piles, Ph.D Tulane University.

Semple R.M. and Gemeinhart J.P. (1981). Stress history approach to analysis of soil resistance to pile driving. 13 th Offshore Technology conference, paper OTC 3969 , pp 165-172.

Stevens R.S., Wiltsie E.A. and Turton T.H. (1982). Evaluating pile drivibility for hard clay, very dense sand and rock. 14 th Offshore Technology conference, paper OTC 4205, pp 465-481.

Vergobbi, P. et Poulet D. (1992). Application des techniques de prévision, d'instrumentation et de contrôle de battage aux ouvrages portuaires. Journées Nationales Génie côtier \& Génie Civil, Nantes, février, 7 p. 